\title{
BETA-LACTAMASE DE ESPECTRO ESTENDIDO: PREVALÊNCIA E COMPARAÇÃO DE MÉTODOS DE SCREENING
}

\author{
ROSÂNGELA CAMINOTTO BARBOSA \\ CLAUDIA MARIA CORREIA E SILVA? \\ SILVIAMICHIKO HIZUKA ${ }^{2}$ \\ EMERSON DANGUY CAVASSIN ${ }^{3}$ \\ MÁRCIA REGINA ECHES PERUGINI ${ }^{4}$
}

\begin{abstract}
BARBOSA, R. C.; SILVA, C. M. C.; HIZUKA, S. M.; CAVASSIN, E. D.; PERUGINI, M. R. E. Beta-lactamase de espectro estendido: prevalência e comparação de métodos de screening. Semina: Ci. Biol. Saúde, Londrina, v. 20/21, n. 2, p. 17-24, jun. 1999/2000.
\end{abstract}

\begin{abstract}
RESUMO: As Beta-lactamases de Espectro Ampliado podem produzir uma oculta mas clinicamente significante, resistência à cefalosporinas de amplo espectro e aztreonam. Estas enzimas são produzidas principalmente por Klebsiella sp e Escherichia coli. Critérios interpretativos modificados para um grupo chave de antibióticos ou o uso de testes especiais de susceptibilidade antimicrobiana devem ser usados para aumentar a sensibilidade de detecção das ESBL. 735 isolados de E. coli e 192 de Klebsiella sp foram estudados para determinar a prevalência de ESBL no Hospital Universitário Regional Norte do Paraná. Os resultados obtidos indicam 2,99\% de E. coli e $36.46 \%$ de Klebsiella sp produtoras de ESBL. Usando um grupo de 156 amostras de E. coli e 74 de Klebsiella sp, comparou-se 3 métodos de triagem para detecção de ESBL: teste de sinergia de duplo disco, disco difusão (ambos realizados com ceftazidima, cefotaxima e aztreonam) e painel de microdiluiçāo MicroScan realizado com ceftazidima, ceftriaxona e cefotaxima). Os resultados deste estudo sugerem que os 3 métodos de triagem podem ser igualmente utilizados por laboratórios clinicos em isolados de E. coli e Klebsiella sp.
\end{abstract}

PALAVRAS-CHAVE: Beta-lactamase; Beta-lactamases de Espectro Ampliado (ESBL); beta-lactâmicos; mecanismos de resistência; Klebsiella sp; Escherichia coli.

\section{INTRODUÇÃO}

Desde a introdução da penicilina $G$ até as cefalosporinas de quarta geração, vem-se observando uma crescente perda de atividade destas drogas, na dependência da pressão seletiva imposta sobre as populações bacterianas (Mendonça, 1996).

A produção de beta-lactamases é o principal mecanismo de resistência das bactérias Gramnegativo, aos agentes beta-lactâmicos (Bush et al., 1995; Livermore, 1995).

Nos últimos 10 anos, tem ocorrido um aumento na prevalência de cepas produtoras de uma betalactamase de espectro estendido (ESBL), de codificação plasmidial, derivada das betalactamases clássicas (TEM-1,TEM-2 e SHV-1). As ESBLs estão incluídas no grupo 2 be de BUSH e possuem ação hidrolítica ampliada a todos os beta- lactâmicos, incluindo cefalosporinas de quarta geração e monobactans, com exceção das cefamicinas e carbapenens. Outra característica fenotípica importante, é que elas permanecem sensiveis a ação de inibidores de beta-lactamases (Philippon,1989; Blatt, 2000; Jacoby, 2000; Livermore, 2000; Silva, 2000).

Estas enzimas ocorrem predominantemente em Klebsiella pneumoniae e Escherichia coli, mas podem também ser encontradas, com menor frequência, em outras espécies de Enterobactérias como Klebsiel/a oxytoca, Enterobacter spp., Proteus spp., Morganella morganii, Salmonella spp.e Serratia marcescens (Thomson,1997; Vercauteren et al.,1997).

As ESBLs foram descritas pela primeira vez, em isolados de Klebsiella na Alemanha em 1983, tendo a primeira grande eclosão ocorrido na França em 1985-87. Cepas com estas enzimas foram relatadas

\footnotetext{
1 Aluna do Curso de Especialização em Análises Clínicas do Departamento de Patologia Aplicada, Legislação e Deontologia (PALD), Centro de Ciências da Saúde (CCS) da Universidade Estadual de Londrina (UEL).

2 Acadêmicas do Curso de Farmácia e Bioquímica da UEL.

${ }^{3}$ Microbiologista do Laboratório de Análises Clínicas do Hospital Universitário Regional Norte do Paraná (HURNP).

${ }^{4}$ Professora Adjunta da disciplina de Microbiologia Clínica do Departamento de Patologia Aplicada Legislação e Deontologia, do Centro de Ciências da Saúde da UEL.
} 
pela primeira vez nos Estados Unidos em 1989-90

Entre os anos de 1988 e 1990 foram detectadas na Espanha os primeiros isolamentos de enterobactérias produtoras de ESBL (Knothe et al., 1983; Livermore, 1995; Sirot et al., 1987; Petit et al., 1990).

Bactérias produtoras de ESBL tem sido isoladas com maior freqüência em amostras provenientes de pacientes hospitalizados, porem também podem ser encontradas em amostras de origem comunitária. Pacientes submetidos a um consumo de antibióticos (principalmente cefalosporinas, em especial ceftazidima) e a cateterização arterial e/ou urinária, possuem maior risco de adquirir infecção ou mesmo colonização por bactérias ESBL. (Silva, 2000).

As beta-lactamases de espectro estendido representam um sério problema clínico nos hospitais. Por ser codificada por plasmídios, esta resistência pode ser transferida facilmente, por conjugação, entre diferentes espécies bacterianas. Além disto, estes plasmídios podem ter, associados, genes de resistência a outros tipos de antimicrobianos. (Silva, 2000).

Os testes de sensibilidade disponiveis atualmente, apresentam limitações na detecção de resistência mediada por estas enzimas (Blatt, 2000; Sader, 1998). Algumas cepas produtoras de ESBL podem mostrar um baixo nivel de resistência que pode não alcançar os "breakpoints" para resistência, preconizados atualmente pelo NCCLS e serem reportadas como sensiveis. Esta falsa sensibilidade pode acarretar falha terapêutica quando infecções causadas por cepas produtoras de ESBL são tratadas com beta-lactâmicos (Blatt, 2000; Sader, 1998; Sader, 2000a; Sader, 2000b).

Vários métodos de triagem para pesquisa de ESBL têm sidos propostos (Jacoby, 1996; NCCLS, 2000; Thomson et al., 1996). Entre estes métodos, tem-se o uso de "breakpoints" modificados, estabelecidos pelo NCCLS (NCCLS, 2000; Thomson et al., 1996), teste de dupla difusão (Jarlier et al., 1988), teste tridimensional (Thomson \& Sanders, 1992) e o método de triagem com fitas ETEST (Silva, 2000; Vercauteren et al., 1997)

Tendo em vista a alta frequência de Escherichia colie Klebsiella sp no HURNP e a alta prevalência de ESBL que tem sido reportada nestes gêneros por diversos autores (Emery \& Weymouth, 1997; Sirot et al., 1992), o presente estudo tem por objetivo, determinar a prevalência de $E S B L$ no HURNP, bem como comparar a habilidade dos métodos de triagem em ágar disco difusão e microdiluição automatizada com "breakpoints" modificados (NCCLS-2000) e teste de dupla difusão, em detectar a expressão desta enzima.

\section{MATERIAIS E MÉTODOS}

\section{Amostras bacterianas:}

Foram incluidas neste estudo, 735 amostras de Escherichia coli e 192 de Klebsiella sp, isoladas de diversos materiais biológicos, obtidos de pacientes internados e atendidos no Hospital Universitário Regional Norte do Paraná, durante o ano de 2000.

Das 927 amostras bacterianas analisadas, 156 Escherichia coli e 77 Klebsiella sp foram selecionadas para serem submetidas aos testes comparativos para pesquisa de ESBL.

\section{Identificação das amostras:}

A identificação em gênero e espécie foi realizada por metodologia manual, segundo orientações do Manual of Clinical Microbiology (Murray et al., 1999), sendo confirmada através de método automatizado Dade Behring MicroScan, utilizando os painéis NC 20 e NUC 1

Após identificadas, as amostras foram estocadas a $-20^{\circ} \mathrm{C}$ em caldo TSB com $30 \%$ de glicerina.

\section{Controle de qualidade:}

Amostras de Escherichia coli ATCC 25922 e ATCC 35218 , foram utilizadas como controle de qualidade neste estudo.

\section{Métodos de detecção de ESBL:}

1- DISCO DIFUSÃO : Os halos de inibição para cefotaxima, ceftazidima e aztreonam, foram medidos e analisados de acordo com os "breakpoints" indicativos da produção de ESBL estabelecidos pelo NCCLS-2000, ou seja, são suspeitas de ESBL as cepas com sensibilidade diminuída, apresentando um halo $=22 \mathrm{~mm}$ para ceftazidima, $=27 \mathrm{~mm}$ para aztreonam e cefotaxima. (Mendes et al., 1998; Blatt, 2000; Aguilera \& Trindade, 2000; NCCLS, 2000).

2- MICRODILUIÇÃO AUTOMATIZADA: A susceptibilidade aos antimicrobianos foi determinada pelo Sistema MicroScan (Dade Behring),usando painéis desidratados para gramnegativo NC 20 e NUC 1, lidos pelo Walkaway 40 e interpretados de acordo com os critérios do NCCLS 2000. A recomendação do NCCLS para a detecção de bactérias suspeitas de produzirem ESBL, inclui que isolados clínicos de Klebsiella spp. e Escherichia coli com MIC aumentados $(==2 \mathrm{ug} / \mathrm{ml})$ para qualquer uma das drogas padronizadas do sistema, ceftazidima, cefotaxima e ceftriaxona, devem ser consideradas suspeitas. 
3- APROXIMAÇÃO DE DISCOS OU DUPLA DIFUSÃO: Em placa de Mueller-Hinton Agar (Difco), foram dispensados discos de ceftazidima $30 \mathrm{ug}$, cefotaxima $30 \mathrm{ug}$ e aztreonam $30 \mathrm{ug}$, ao redor de um disco central de amoxicilina $20 \mathrm{ug} /$ ácido clavulânico 10 ug, distantes deste, $20 \mathrm{~mm}$ de bordo a bordo. Considerou-se teste positivo para ESBL, sinergia positiva, a presença de ampliação e/ou distorção do halo de inibição de um ou mais antimicrobianos testados ou, ainda, o aparecimento de uma terceira zona irregular de inibição entre o disco de amoxicilina+ácido clavulânico e o disco de uma das drogas betalactâmicas ("ghost-zone").

4- Fitas ETEST: Esta metodologia foi utilizada como referência ouro, nos casos em que houve discordância de resultados. Uma suspensão bacteriana, com turbidez correspondente à 0.5 da escala de McFarland, foi semeada em uma placa de Mueller-Hinton Ágar. Após um intervalo de 15 minutos, foi dispensada a fita de ETEST, que apresenta gradientes de concentrações estáveis de ceftazidima $(0,5 \mathrm{ug} / \mathrm{ml}$ a $32 \mathrm{ug} / \mathrm{ml})$ em uma das extremidades e ceftazidima $(0,125$ $\mathrm{ug} / \mathrm{ml}$ a $8 \mathrm{ug} / \mathrm{ml}$ ) associada a uma concentração fixa de ácido clavulânico $(2 \mathrm{ug} / \mathrm{ml})$ na extremidade oposta. Conforme instruções do fabricante, a MIC fol determinada como sendo a primeira concentração acima da intersecção entre a fita de ETEST e a zona eliptica de inibição de crescimento bacteriano. A amostra foi considerada produtora de ESBL, quando ocorreu uma redução da MIC de ceftazidima, 2,5 diluições logaritmicas na presença de ácido clavulânico em comparação com a MIC de ceftazidima. Em casos onde ambas as MiC apresentaram valores abaixo dos gradientes de antimicrobianos presentes na fita, a redução foi considerada zero.

\section{RESULTADOS}

Das 735 amostras de Escherichia coli testadas, $22(2,99 \%)$ foram positivas para ESBL por um ou mais dos métodos de triagem (Figura 1). Das 192 Klebsiella sp analisadas, $70(36,46 \%)$ se mostraram produtoras da enzima (Figura 2 ).

Considerando as 156 amostras de Escherichia coli analisadas comparativamente pelos métodos de detecção de ESBL, 12 (7,69 \%) foram positivas pelo método de disco difusão e $9(5,76 \%)$ pela aproximação de discos. A microdiluição automatizada MicroScan foi realizada em 75 destas amostras, das quais somente $5(6,66 \%)$ foram consideradas positivas para ESBL (Figura 3 ).

Das 74 amostras de Klebsiella sp submetidas aos testes comparativos, $46(62,16 \%)$ foram positivas

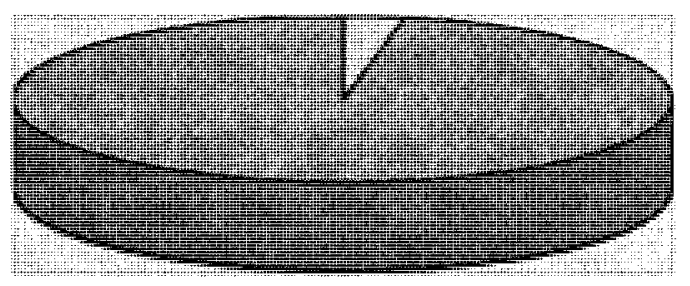

\section{$\square$ ESBL 图 não ESBL}

Figura 1- Prevalência de ESBL entre 735 amostras de E. coli

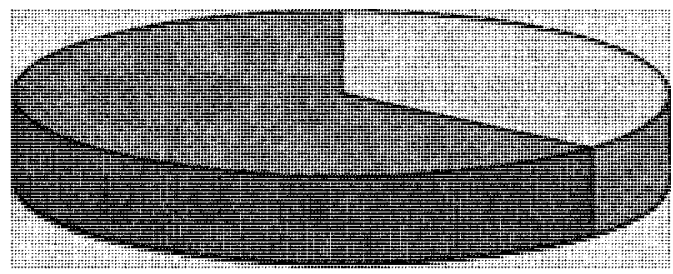

GSBL

圆 não ESBL

Figura 2 - Prevalência de ESBL entre 192 amostras de Klebsiella sp. 
por disco difusão e $42(56,75 \%)$ pela aproximacão de discos. Destas amostras, 25 foram testadas por microdiluicão automatizada, das quais $13(52 \%)$ foram consideradas produtoras da enzima (Figura 4). Estes resultados não representam prevalência, uma vez que os microorganismos não foram coletados de maneira consecutiva.

Comparando-se os métodos de disco difusão e microdiluicão automatizada, observou-se concordância em 100\% das amostras de Escherichia coli (Tabela 1) e de Klebsiella sp (Tabela 2).

Com relação aos métodos de disco difusão e aproximacão de discos (DDS), notou-se concordância absoluta entre as amostras negativas para ESBL por ambos os métodos, porém houve discordância em 3 das amostras positivas de Escherichia coli (Tabela 3) e 4 das amostras de Klebsiella sp (Tabela 4).

Comparando o teste de microdiluicão automatizada com a dupla difusão (DDS), embora todas as amostras negativas das 2 bactérias apresentem concordância de $100 \%$ entre os dois métodos, observou-se discordância em 3 amostras de Escherichia coli (Tabela 5) e 2 de Klebsiella sp (Tabela 6) na análise dos resultados.

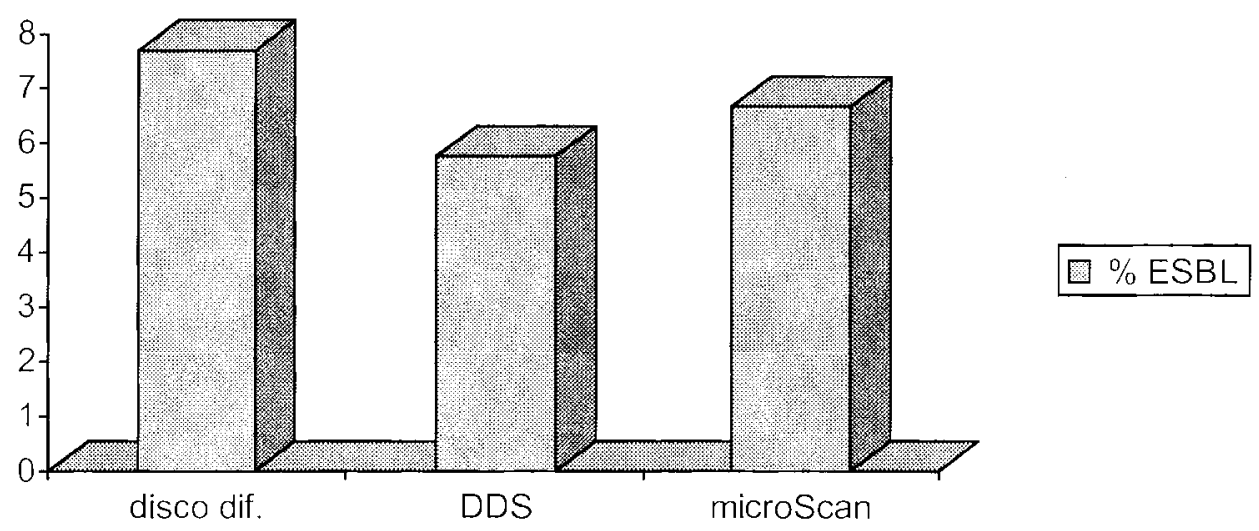

Figura 3 - Positividade de ESBL pelos métodos de triagem em 156 amostras de E. coli

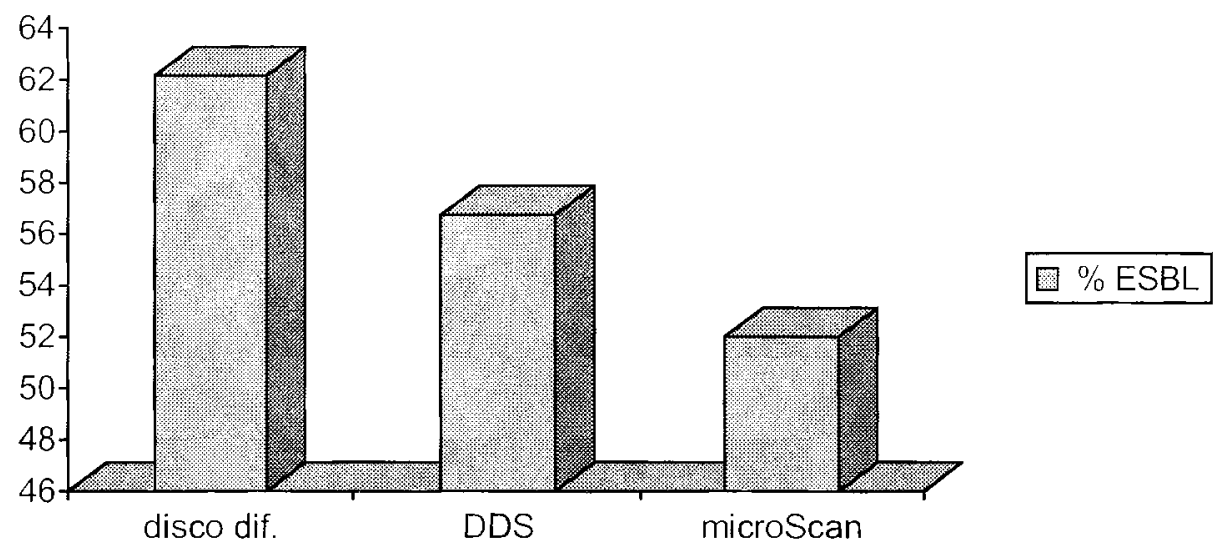

Figura 4 - Positividade de ESBL pelos métodos de triagem em 74 amostras de Klebsiel/a sp 
Tabela 1 - Comparação entre os métodos de disco difusão e microdiluição em 75 amostras de E. coli.

\begin{tabular}{llcl}
\hline & & DISCO DIFUSÃO & \\
\hline MICRODILUIÇÃO & + & - & TOTAL \\
+ & 05 & 00 & 05 \\
- & 00 & 70 & 70 \\
TOTAL & 05 & 70 & 75 \\
\hline
\end{tabular}

Tabela 2 - Comparação entre os métodos de disco difusão e microdiluição em 25 amostras de Klebsiella sp.

\begin{tabular}{llcl}
\hline & & DISCO DIFUSÃO & \\
\hline MICRODILUIÇÃO & + & - & TOTAL \\
+ & 13 & 00 & 13 \\
- & 00 & 12 & 12 \\
TOTAL & 13 & 12 & 25 \\
\hline
\end{tabular}

Tabela 3 - Comparação entre os métodos de disco difusão e aproximação de discos (DDS) em 156 amostras de E. coli

\begin{tabular}{llll}
\hline & \multicolumn{3}{c}{ DDS } \\
\hline DISCO DIFUSÃO & + & - & TOTAL \\
+ & 09 & 03 & 12 \\
- & 00 & 144 & 144 \\
TOTAL & 09 & 147 & 156 \\
\hline
\end{tabular}

Tabela 4 - Comparação entre os métodos de disco difusão e aproximação de discos(DDS) em 74 amostras de Klebsiella sp.

\begin{tabular}{llll}
\hline & & DDS & \\
\hline DISCO DIFUSÃO & + & - & TOTAL \\
+ & 42 & 04 & 46 \\
- & 00 & 28 & 28 \\
TOTAL & 42 & 32 & 74 \\
\hline
\end{tabular}

Tabela 5 - Comparação entre os métodos de microdiluição e aproximação de discos (DDS) em 75 amostras de E. coli.

\begin{tabular}{llll}
\hline & \multicolumn{3}{c}{ DDS } \\
\hline MICRODILUIÇÃO & + & - & TOTAL \\
+ & 02 & 03 & 05 \\
- & 00 & 70 & 70 \\
TOTAL & 02 & 73 & 75 \\
\hline
\end{tabular}

Tabela 6 - Comparação entre os métodos de microdiluição aproximação de discos (DDS) em amostras de Klebsiella sp.

\begin{tabular}{llll}
\hline & \multicolumn{2}{c}{ DDS } \\
\hline MICRODILUIÇÃO & + & - & TOTAL \\
+ & 11 & 02 & 13 \\
- & 00 & 12 & 12 \\
TOTAL & 11 & 14 & 25 \\
\hline
\end{tabular}


quantitativamente mensurado (Reis, 1999), além de ser influenciado pela distância entre os discos. Segundo alguns autores (Moland et al.,1998), utilizando o "breakpoint" $=2 \mathrm{ug} / \mathrm{ml}$ recomendado pelo NCCLS para detectar a maioria das ESBL (82 a $91 \%$ ), o MicroScan pode incluir nesta detecção, outras enzimas que não ESBL, como AmpC betalactamase. No trabalho, os autores recomendam a utilização de outros valores como um "breakpoint" de $=4 \mathrm{ug} / \mathrm{ml}$ para ceftazidima, a fim de eliminar possiveis falsos positivos.

Devido à sua acurácia, o ETEST seria o método mais indicado para a pesquisa de $E S B L$, porém em virtude do alto custo das fitas, o uso de "breakpoints" modificados para os testes de triagem, disco difusão e determinação da MIC, embora não sejam confirmatórios da produção de ESBL, representam uma opção bastante prática para os laboratórios de rotina, pois não implica em mudanças na realização dos testes (Reis, 1999). Em nosso trabalho, os métodos utilizados apresentaram boa concordância, em relação aos substratos testados, podendo ser implantados em laboratórios de rotina de nossa região, como triagem na detecção de amostras suspeitas de ESBL.

Considerando a alta prevalência de ESBL e as possiveis falhas terapêuticas, è essencial o trabalho conjunto do laboratório e do clínico em estudos epidemiológicos e identificação precoce destas cepas, a fim de orientar as medidas a serem implementadas pelas comissões de controle de infecção hospitalar, como no uso indiscriminado de antibióticos, principalmente se atentarmos para o fato de que muitos genes ESBL localizam-se em plasmídios de ampla multi-resistência, os quais também codificam resistência para outros antibióticos (Aguilera \& Trindade, 2000, Jacoby, 2000, Patterson, 2000).

BARBOSA, R. C.; SILVA, C. M. C.; HIZUKA, S. M.; CAVASSIN, E. D.; PERUGINI, M. R. E. Extendedspectrum Beta-lactamases: prevalênce and comparison of screening for detection. Semina: Ci. Biol. Saúde, Londrina, v. 20/21, n. 2, p. 17-24, jun. 1999/2000.

ABSTRACT: The Extended Spectrum Beta-lactamases may produce hidden but clinically significant resistance to expanded-spectrum cephalosporins and aztreonam. This enzimes are produced mainly by Klebsiella and Escherichia coli. Modified interpretive criteria for key antibiotics or the use of special antimicrobial susceptibility tests should be used to increase the sensitivity of ESBL detection. 735 isolates of Escherichia coli and 192 of klebsiella sp were studied for determine the prevalence of ESBL in HURNP. The results obtained indicate $2,99 \%$ of E. coli and $36,46 \%$ of Klebsiella sp that encode for resistance to broad-spectrum beta-lactam antibiotics. Using a set of 156 strains of E. coli and 74 of Klebsiella sp, we compared three screening methods for ESBL detection: a double-disk synergy test, disk diffusion (both test were performed with ceftazidime, cefotaxime and aztreonam) and MicroScan microdilution pane/s (performed with ceftazidime and ceftriaxone e/ou cefotaxime). The results of this study suggest that three screening methods can be equaly used by clinical laboratories as na indicator of extended-spectrum beta-lactamases, in isolates of E. coli and Klebsiella sp.

KEY WORDS: Beta-lactamase; Extended Spectrum Beta-lactamases (ESBL); Beta-lactam; resistance mecanisms; Klebsiella sp; Escherichia coli.

\section{REFERÊNCIAS BIBLIOGRÁFICAS}

AGUILERA, L.; TRINDADE, N. Informações sobre Betalactamases de espectro estendido, induzidas e outras informações. LAES \& HAES, v.39, p. 244-248, 2000.

BLATT, J. M. Mecanismo de resistência e detecção das Betalactamases de espectro ampliado. Newslab, v. 40, p. 8697, 2000.

BUSH, K.; JACOBY, G. A.; MEDEIROS, A. A. A Functiona classification scheme for Beta-lactamases and its correlation with molecular structure. Antimicrobial Agents and Chemotherapy, p. 1211-1233, June, 1995.

EMERY, C. L. ; WEYMOUTH, L. Detection and clinicalsignificance of extended-spectrum beta-lactamases in a tertiary-care medical center. J. Clin. Microbiol., v.35 p. 2061-2067,1997.
JACOBY, G. A.; HAN, P. Detection of extended-spectrum betalactamases in clinical isolates of Kiebsiella pneumoniae and Escherichia coli. Jor. Clin. Microbiol., v.34, p. 908-911, 1996.

JACOBY, G. A. Desenvolvimento da resistência em patógenos gram-negativos - Beta-lactamases de amplo espectro. Hospital Practice., p. 14-21, jul. 2000.

JACOBY, G. A., and MEDEIROS, A. More extended-spectrum beta-lactamases. Antimicrob. Agents Chemother., v. 35, p. $1697-1704,1991$

JARLIER, V.; NOCOLAS, M. H.; FOURNIER, G.; PHILIPPON, A. Extended broad-spectrum Beta-lactamases conferring 
transferable resistance to newer Beta-lactam agents in Enterobacteriaceae: hospital prevalence and susceptibility patterns. Ver. Infect. Dis. v.10, p. 867-878, 1988

KNOTHE. H.; SHAH P.; KREMERY V.: ANTAL M.; MITSHASHIS Transferable resistance to cefotaxime, cefoxitin, cefamandole, and cefuroxime in clinical isolates of Klebsiella pneumoniae and Serratia marcescens. Infection. v.11, p. 315-317, 1983.

LIVERMORE, D. M.. Beta-lactamases in Laboratory and Clinical Resistance. Clinical Microbiology Reviews, p. 557-584 Oct. 1995

MENDES, C. M. F.; MIMICA, I. M.; MIMICA, L. M. J. Manual de microbiologia clinica aplicada ao controle de infecçáo hospitalar. [S.I.: s.n.], 1998. p. 46-61

MENDONÇA, J. S. Conquistas Recentes da Moderna Antibioticoterapia. Moderna Antibioticoterapia., v. 1, n. 3, p. $1-8,1996$

MOLAND, E. S.; SANDERS, C. C.: THOMSON, K. S. Can results obtained with commercially available microscan microdilution panels serve as an indicator of Beta-lactamase production among Escherichia coli and Klebsiella isolates with hidden resistance to expanded-spectrum cephalosporins and aztreonam?. Journal of Clinical Microbiology. v. 36 p. 2575-2579, Sept. 1998.

MURRAY, P. R.; BARAN, E. J.; PFALLER, M. A.; TENOVER, F. C.; YOLKEM, R. H. Manual of Clinical Microbiology. 7. ed. [ S.I.] American Society of Microbiology, , 1999

NATIONAL COMMITTEE FOR CLINICAL LABORATORY STANDARDS ESBL Working Group. Arnual meeting, subcommittee on antimicrobial susceptibility testing. [S.I.: s.n.], 1997

Methods diluition antimicrobial susceptibility tests for bacteria that grow aerobic. Approved standard M 100 $\$ 10,2000$

PATTERSON, J. E. Beta-lactamases de amplo espectro: intervençōes bem sucedidas em casos de resistência de gram-negativos, tratamento, controle e prevenção. Hospital Practice, p.22-27, jul., 2000.

PETIT, A.: GERBAUD G.; SIROT D.; COURVALIN P.; SIROT J. Molecular epideniology of TEM-3 (CTX-1) beta-lactamase. Antimicrob. Agents Chemother., v. 34, p. 219-224, 1990.

PHILIPPON, A.; LABIA R.; JACOBY G. Extended-spectrum beta-lactamases. Antimicrob. Agents Chemother. 1989.

PRAGAI, Z.; KOCZIAN, Z.; NAGY, E. Characterization of the extended-spectrum beta-lactamases and determination of the antibiotic susceptibilities of Klebsiella pneumoniae isolates in Hungary. J Antimicrob. Chemother, v. 42, p. $401-403,1998$

REIS, A . O . Avaliação dos testes laboratoriais para detecção de amostras de Klebsiella pneumoniae produtoras de Beta-lactamases de espectro ampliado. 1999. Tese (Mestrado em Doenças Infecciosas e Parasitárias) - Escola Paulista de Medicina, Universidade Federal de São Paulo.

SADER, H. Extend-spectrum beta-lactamases: a potent antimicrobiol resistance mechanism that is spreading rapidly. Publicacion del comité de microbiologia clinica de la API, 1998.

SADER. H. S. Principais problemas de resistência bacteriana encontrados pelo Programa SENTRY no Brasil. SENTRY News, v. 2. p. 1-7, 2000a

Principais problemas de resistência bacteriana encontrados pelo Programa Sentry no Brasil. SENTRY News, v.1, p. 3, 2000 b.

SILVA, C. H. P. M. Beta-lactamase de espectro estendido: definições, importância clinica e detecção laboratorial Revista Brasileira de Análises Clinicas, v. 32, n.3, p. $215-219,2000$

; SALVINO, C. R. Importância do recorihecimento das enterobactérias hospitalares produtoras de Betalactamases de espectro estendido (ESBL) e suas implicaçōes terapêuticas. News Lab., v. 41, p. 104-112, 2000 .

SIROT, D.; SIROT, J.; LABIA, R.; MORAND, A.; COURVALIN, P.; DARFEUILLE, M. A.; PERROUX, R.; CLUNZEL, R. Transferable resistance to third-generation cephalosporins in clinical isolates of Klebsiella pneumoniae: identification of CTX-1, a novel beta-lactamase. J. Antimicrob. Chemother., v. 20, p. 323-334, 1987

THOMSON, K. S. New plasmid-mediated beta-lactamases of gram-negative pathogens: clinical and laboratory implications. Dade MicroScan Inc., 1997.

THOMSON, K. S.; PREVAN, A. M.; SANDERS, C. C. Novel plasmid-mediated Beta-lactamases in Enterobacteriaceae: emerging problems for new Beta-lactam antibiotics. Current Clinical Topics in Infectious Diseases, v. 16, p. 151-163 1996.

THOMSON, K. S.; SANDERS, C. C. Detection of extendedspectrum Beta-lactamases in members of the family Enterobacteriaceae: comparison of the double-disk and three-dimensiona! test. Antimicrob Agents Chemother, v. 36, p.1877-1882, 1992.

VERCAUTEREN, E; DESCHEEEMAEKER, P.; IEVEN, M; SANDERS, C. C.; GOOSSENS, H. Comparison of screening methods for detection of extended-spectrum Betalactamases and their prevalence among blood isolates of Escherichia coli and Klebsiella spp. in a Belgian Teaching Hospital. Journal of Clinical Microbiology. v.35, n. 9, p. 2191-2197, 1997 